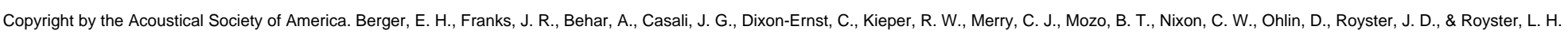

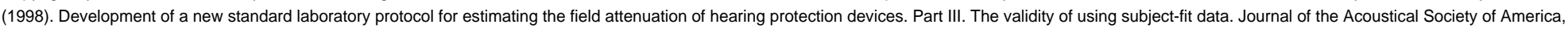
103(2), 665-672. doi: 10.1121/1.423236

\title{
Development of a new standard laboratory protocol for estimating the field attenuation of hearing protection devices. Part III. ${ }^{\text {a) }}$ The validity of using subject-fit data ${ }^{\text {b) }}$
}

\author{
Elliott H. Berger \\ E-A-R/Aearo Company, Indianapolis, Indiana 46268 \\ John R. Franks \\ National Institute for Occupational Safety and Health, Cincinnati, Ohio 45226 \\ Alberto Behar \\ Behar Noise Control, Scarborough, Ontario M1M 2X8, Canada \\ John G. Casali \\ Virginia Tech, Blacksburg, Viriginia 24061
}

Christine Dixon-Ernst

ALCOA, Pittsburgh, Pennsylvania 15219

Ronald W. Kieper

E-A-R/Aearo Company, Indianapolis, Indiana 46268

Carol J. Merry

National Institute for Occupational Safety and Health, Cincinnati, Ohio 45226

Ben T. Mozo

U. S. Army Aeromedical Research Laboratory, Fort Rucker, Alabama 36330

Charles W. Nixon

Systems Research Laboratories, Wright-Patterson Air Force Base, Ohio 45433

Doug Ohlin

U. S. Army Center for Health Promotion and Preventive Medicine, Aberdeen Proving Ground, Maryland 21010

Julia Doswell Royster

Environmental Noise Consultants, Inc., Raleigh, North Carolina 27622

Larry H. Royster

North Carolina State University, Raleigh, North Carolina 27695

(Received 13 May 1997; accepted for publication 19 September 1997)

The mandate of ASA Working Group S12/WG11 has been to develop "laboratory and/or field procedure(s) that yield useful estimates of field performance" of hearing protection devices (HPDs). A real-ear attenuation at threshold procedure was selected, devised, tested via an interlaboratory study, and incorporated into a draft standard that was approved in 1997 [J. D. Royster et al., "Development of a new standard laboratory protocol for estimating the field attenuation of hearing protection devices. Part I. Research of Working Group 11, Accredited Standards Committee S12, Noise," J. Acoust. Soc. Am. 99, 1506-1526 (1996); ANSI S12.6-1997, "American National Standard Methods for Measuring Real-Ear Attenuation of Hearing Protectors" (American National Standards Institute, New York, 1997)]. The real-world estimation procedure utilizes a subject-fit methodology with listeners who are audiometrically proficient, but inexperienced in the use of HPDs. A key factor in the decision to utilize the subject-fit method was an evaluation of the representativeness of the laboratory data vis-à-vis attenuation values achieved by workers in practice. Twenty-two field studies were reviewed to develop a data base for comparison purposes. Results indicated that laboratory subject-fit attenuation values were typically equivalent to or greater than the field attenuation values, and yielded a better estimate of those values than did

\footnotetext{
a) This paper is the last of three parts of a body of work that represents the research and analyses of S12/WG11 in conjunction with the development of ANSI S12.6-1997. Part I appeared in 1996 in J. Acoust. Soc. Am. 99, 1506-1526 (1996). It referenced two succeeding parts, one of which Part II is still in press and hence will appear out of chronological order. Additionally the advance citation of this paper in Part I, listed the first two authors in the reverse order from that which appears above.

b) "Selected research articles" are ones chosen occasionally by the Editor-in-Chief that are judged (a) to have a subject of wide acoustical interest, and (b) to be written for understanding by broad acoustical readership.
} 
experimenter-fit or experimenter-supervised fit types of results. Recent data which are discussed in the paper, but which were not available at the time of the original analyses, confirm the findings. (C) 1998 Acoustical Society of America. [S0001-4966(98)03001-X]

PACS numbers: 43.10.Ln, 43.50.Hg, 43.66.Vt, 43.15.+s [GAD]

\section{INTRODUCTION}

A quantity of fundamental concern to the understanding and description of the performance of hearing protection devices (HPDs) is the attenuation provided by such devices under conditions of actual use. Since the mid-1970's, studies have been published with increasing regularity (Berger $e t$ al., 1996) indicating that the standardized laboratory test procedures utilized in North America do a very poor job of predicting such performance (ANSI S3.19-1974, ANSI S12.61984). This discrepancy between laboratory and real-world data is especially troubling considering the importance that many hearing protector purchasers and users ascribe to published attenuation values. As a result, Accredited Standards Committee S12, Noise, assigned its Working Group 11, "Hearing Protector Attenuation and Performance," the task of developing a procedure that would yield useful estimates of "achievable field performance" i.e., the noise reduction that properly trained and motivated workers receive from wearing their hearing protectors in occupational settings. Such results were defined as among the higher values of attenuation attained by groups of informed users in wellmanaged and well-supervised industrial and military hearing conservation programs.

This paper describes analyses conducted by the members of Working Group 11 to evaluate the suitability of a proposed standardized laboratory test procedure for measuring real-ear attenuation at threshold (REAT) in a manner that estimates achievable field performance. A prior paper described an interlaboratory comparison study undertaken by Working Group 11 that was used as the basis for the development and evaluation of the proposed protocol (Royster et al., 1996). The reader is referred to that report and to the final approved standard that was developed as the outcome of the Working Group's efforts (ANSI S12.6-1997) for the rationale behind the experiments, a description of the overall work effort, the details of the test procedures, and a presentation of the actual results of the interlaboratory study. This paper will focus on a comparison of the interlaboratory test results to available field studies, in order to draw inferences about the degree to which the laboratory data can appropriately be used to predict field results.

The Working Group's interlaboratory protocol involved two distinctly different methods, an informed user-fit and a subject-fit approach. The latter procedure involved test subjects who were audiometrically proficient, but naive in the use of hearing protection. Prior research had suggested that the subject-fit method would provide better field estimates (Berger, 1988; Casali and Epps, 1986), but the informed user-fit (later somewhat modified and implemented as an experimenter-supervised fit in the final approved standard) was also included in the interlaboratory protocol because of the concern that the subject-fit might lead to large inter- and intralaboratory variability and thus be unsuitable for standardization. However, the outcome of the experiments and subsequent analyses failed to justify such concerns, instead demonstrating that the subject-fit method was even preferable in terms of reproducibility (Royster et al., 1996). With this in mind, as well as the fact that the subject-fit data had already been shown to predict the approximate upper bound estimate of field performance, and that experimentersupervised fitting only diminished the usefulness of the prediction, the decision was made to focus attention on the subject-fit data for the analyses in this report.

\section{METHODS}

\section{A. The laboratory data sample}

The HPDs which were selected for the interlaboratory study, and hence for the real-world comparison, are described in Sec. II C and Fig. 1 of Royster et al. (1996). They include the Aearo Company E-A- ${ }^{\circledR}$ Classic foam earplug, the PlasMed, Inc. V-51R premolded earplugs (5 sizes), the Willson Safety Products EP100 premolded earplugs (2 sizes), and the Bilsom UF-1 earmuffs. The devices were selected because they were products for which the greatest amount of real-world data were available in the literature, because of their popularity in the marketplace at the time of the study, and because they represented a diverse range of product types with a focus on earplugs, which were the type of hearing protector that the Working Group had deemed provided a greater real-world estimation problem than did earmuffs.

The laboratory-based attenuation values used in the following analyses are the average of each test subjects' two trials in the Subject-Fit 1 test session, and their two trials in the Subject-Fit 2 test session, from the interlaboratory study as reported in Table II of Royster et al. (1996). This provided a single attenuation value for each of 24 subjects at each of four laboratories, based on four attenuation measurements per subject. In this paper, trials were averaged together as in the original analyses by Royster et al. Furthermore, sessions were also averaged together since the ANOVAs indicated no effect of practice, i.e., no difference between the Subject-Fit 1 and Subject-Fit 2 test sessions. And finally, the data were also averaged together across the four laboratories since an ANOVA involving only the subject-fit data indicated no overall laboratory effect, albeit with a significant effect for a few cases-1000 and $2000 \mathrm{~Hz}$ for the Bilsom earmuff and $1000 \mathrm{~Hz}$ for the E-A-R Plug, due to lower attenuation values from one laboratory. However, the interlaboratory differences between subject-fit data for the EP100 and V-51R earplug were not significant at any frequency. Therefore all of the subject-fit data were pooled across 96 subjects with four trials per subject to create a benchmark 
TABLE I. E-A-R classic foam earplug. Mean subject-fit (SF) data from the interlaboratory study, manufacturer's labeled values, and 16 real-world studies. $N$ is number of subjects, SD is standard deviation, shaded values are significantly less than Interlab values at $p<0.05$, and underlined values are significantly greater at $p<0.05$.

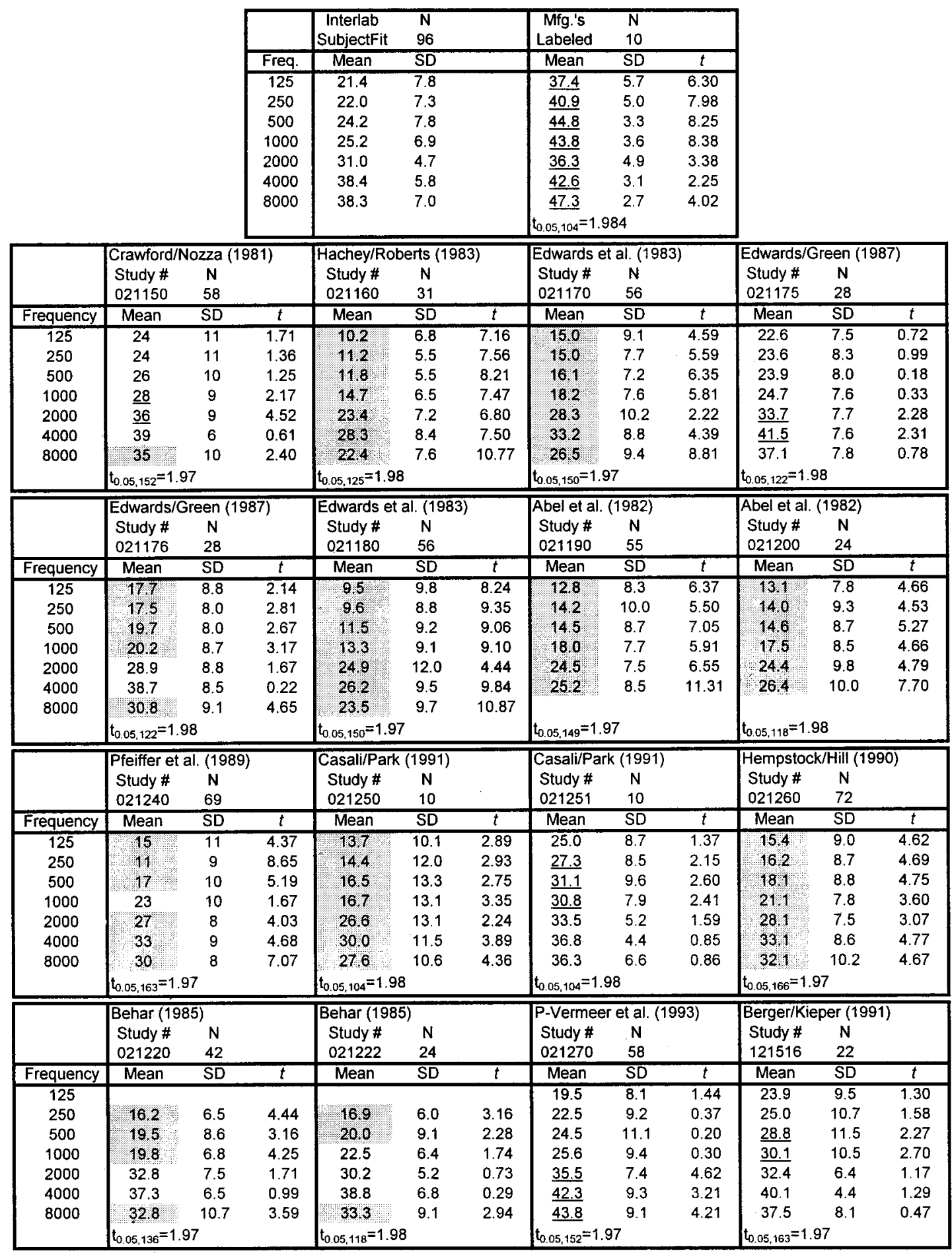

against which the real-world mean attenuations and standard deviations of attenuations could be compared. These averaged values, across practice and across laboratory, are reported in Tables I-IV of this report, in the cells labeled "Interlab Subject Fit.",

Also reported in Tables I through IV are the manufacturers' published values based upon testing according to the experimenter-fit protocol of ANSI S3.19 as interpreted by the U. S. Environmental Protection Agency (EPA, 1979). The EPA's specific implementation does not use the first step of the S3.19-specified experimenter fit (namely, a subject fit of the device), thereby causing the fitting to become a purely experimenter-controlled procedure. In effect, the current EPA interpretation and test lab practices utilize the subject as 
TABLE II. V-51R earplug. Mean subject-fit (SF) data from the interlaboratory study, manufacturer's labeled values, and 5 real-world studies. $N$ is number of subjects, SD is standard deviation, shaded values are significantly less than Interlab values at $p<0.05$, and underlined values are significantly greater at $p<0.05$.

\begin{tabular}{|c|c|c|c|c|c|c|}
\hline & $\begin{array}{c}\text { Interlab } \\
\text { Subject Fit }\end{array}$ & $\begin{array}{l}N \\
96 \\
\end{array}$ & & $\begin{array}{l}\text { Mfg.'s } \\
\text { Labeled }\end{array}$ & $\begin{array}{l}N \\
10 \\
\end{array}$ & \\
\hline Frequency & Mean & $\overline{S D}$ & & Mean & SD & $T$ \\
\hline 125 & 11.5 & 10.4 & & $\underline{20}$ & 2 & 2.57 \\
\hline 250 & 10.9 & 10.0 & & $\underline{22}$ & 2 & 3.49 \\
\hline 500 & 11.6 & 10.0 & & $\underline{24}$ & 2 & 3.90 \\
\hline 1000 & 14.1 & 10.2 & & $\underline{28}$ & 2 & 4.28 \\
\hline 2000 & 21.3 & 9.9 & & $\underline{34}$ & 2 & 4.03 \\
\hline 4000 & 22.8 & 7.9 & & $\overline{37}$ & 3 & 5.62 \\
\hline \multirow[t]{5}{*}{8000} & 18.6 & 11.2 & & $\underline{37}$ & 3 & 5.16 \\
\hline & & & & \multicolumn{3}{|l|}{$t_{0.05,104}=1.98$} \\
\hline & \multicolumn{3}{|c|}{ Royster et al. (1991) } & \multicolumn{3}{|c|}{ Abel et al. (1982) } \\
\hline & Study \# & $\mathrm{N}$ & & Study \# & $\mathrm{N}$ & \\
\hline & 017222 & 12 & & 017802 & 20 & \\
\hline Frequency & Mean & SD & $\bar{t}$ & Mean & SD & $\bar{t}$ \\
\hline 125 & & & & 10.8 & 10.2 & 0.27 \\
\hline 250 & & & & 13.3 & 10.1 & 0.97 \\
\hline 500 & 14.6 & 14.5 & 0.93 & 12.3 & 9.5 & 0.29 \\
\hline 1000 & 14.8 & 11.5 & 0.22 & 10.5 & 9.3 & 1.46 \\
\hline 2000 & 18.8 & 8.7 & 0.83 & 13.9 & 8.6 & 3.11 \\
\hline 4000 & 22.0 & 10.3 & 0.32 & 15.6 & 9.4 & 3.59 \\
\hline \multirow{5}{*}{8000} & & & & & & \\
\hline & $t_{0.05,106}=1.98$ & & & $t_{0.05,114}=1.97$ & & \\
\hline & \multicolumn{3}{|c|}{ Edwards et al. (1978) } & \multicolumn{3}{|l|}{ Fleming (1980) } \\
\hline & Study \# & $\mathrm{N}$ & & Study \# & $\mathrm{N}$ & \\
\hline & 019901 & 84 & & 019902 & 9 & \\
\hline Frequency & Mean & $\overline{S D}$ & $\bar{t}$ & Mean & SD & $t$ \\
\hline 125 & 9.0 & 11.0 & 1.57 & 8.6 & 5.4 & 0.82 \\
\hline 250 & 9.0 & 10.0 & 1.27 & 9.4 & 7.1 & 0.44 \\
\hline 500 & 9.0 & 11.0 & 1.66 & 11.4 & 8.7 & 0.06 \\
\hline 1000 & 13.0 & 11.0 & 0.70 & 15.9 & 6.3 & 0.52 \\
\hline 2000 & 20.5 & 14.0 & 0.45 & 21.9 & 9.9 & 0.17 \\
\hline 4000 & 20.0 & 11.0 & 1.98 & 21.3 & 8.4 & 0.54 \\
\hline \multirow[t]{5}{*}{8000} & 14.0 & 12.0 & 2.66 & 18.4 & 12.6 & 0.05 \\
\hline & \multicolumn{3}{|l|}{$t_{0.05,178}=1.97$} & \multicolumn{3}{|l|}{$t_{0.05,104}=1.98$} \\
\hline & \multicolumn{3}{|c|}{ Padilla (1976) } & & & \\
\hline & Study \# & $\mathrm{N}$ & & & & \\
\hline & 019903 & 183 & & & & \\
\hline Frequency & Mean & SD & $\bar{t}$ & & & \\
\hline \multirow{2}{*}{\multicolumn{7}{|c|}{$\begin{array}{l}125 \\
250\end{array}$}} \\
\hline & & & & & & \\
\hline \multirow{2}{*}{\multicolumn{7}{|c|}{$\begin{array}{c}500 \\
1000\end{array}$}} \\
\hline & & & & & & \\
\hline \multicolumn{7}{|l|}{2000} \\
\hline \multicolumn{7}{|l|}{4000} \\
\hline & \multicolumn{6}{|l|}{$t_{0.5111}=1.97$} \\
\hline
\end{tabular}

though she or he were a test fixture to which the experimenter applies the HPD being tested. This type of manufacturers' data represents the information most commonly available to customers in North America today for purposes of specifying and selecting HPDs.

\section{B. The real-world data sample}

The first reported data on field performance of HPDs were published by Reagan in 1975. Since then, at least 21 additional studies of which the authors are aware have become available worldwide (Abel et al., 1982; Behar, 1985; Berger and Kieper, 1991; Casali and Park, 1991; Chung et al., 1983; Crawford and Nozza, 1981; Durkt, 1993; Edwards et al., 1983; Edwards and Green, 1987; Edwards et al., 1978; Fleming, 1980; Goff and Blank, 1984; Hachey
TABLE III. Wilson EP-100 earplug. Mean subject-fit (SF) data from the interlaboratory study, manufacturer's labeled values, and 5 real-world studies. $N$ is number of subjects, $\mathrm{SD}$ is standard deviation, shaded values are significantly less than Interlab values at $p<0.05$, and underlined values are significantly greater at $p<0.05$.

\begin{tabular}{|c|c|c|c|c|c|c|}
\hline & $\begin{array}{c}\text { Interlab } \\
\text { Subject Fit }\end{array}$ & $\begin{array}{l}N \\
96\end{array}$ & & $\begin{array}{c}\text { Mfg.'s } \\
\text { Labeled }\end{array}$ & $\begin{array}{c}N \\
10\end{array}$ & \\
\hline Frequency & Mean & SD & & Mean & SD & $t$ \\
\hline 125 & 14.7 & 11.7 & & 27 & 3.9 & 3.29 \\
\hline 250 & 14.5 & 11.6 & & 29 & 2.9 & 3.92 \\
\hline 500 & 15.4 & 12.5 & & 31 & 3.0 & 3.92 \\
\hline 1000 & 17.5 & 11.1 & & $\overline{33}$ & 3.0 & 4.38 \\
\hline 2000 & 24.4 & 10.2 & & $\underline{37}$ & 4.0 & 3.86 \\
\hline 4000 & 30.1 & 11.1 & & $\underline{45}$ & 3.6 & 4.21 \\
\hline \multirow[t]{3}{*}{8000} & 27.0 & 14.0 & & $t_{0.05 .104}=1.98$ & $t_{0.05 .104}=1.98$ & 2.02 \\
\hline & \multicolumn{3}{|c|}{ Crawford/Nozza (1981) } & \multicolumn{3}{|c|}{ Edwards et al. (1978) } \\
\hline & $\begin{array}{l}\text { Study \# } \\
018901\end{array}$ & $\begin{array}{l}N \\
22\end{array}$ & & $\begin{array}{l}\text { Study \# } \\
018903\end{array}$ & $\begin{array}{l}N \\
28\end{array}$ & \\
\hline Frequency & Mean & SD & $t$ & Mean & SD & $t$ \\
\hline 125 & 8 & 12 & 2.41 & 5 & 8 & 4.11 \\
\hline 250 & 8. & 12 & 2.36 & 4 & 8 & 4.48 \\
\hline 500 & 10 & 11 & 1.87 & 5 & 8 & 4.15 \\
\hline 1000 & 12 & 13 & 2.03 & 6 & 8 & 5.10 \\
\hline 2000 & 22 & 15 & 0.90 & 13 & 13 & 4.88 \\
\hline 4000 & $20 \%$ & 11 & 3.86 & 18. & 10 & 5.18 \\
\hline \multirow[t]{5}{*}{8000} & 14.9 & 12 & 4.03 & 9 & 12 & 6.17 \\
\hline & \multicolumn{3}{|l|}{$t_{0.05 .116}=1.97$} & \multicolumn{3}{|l|}{$t_{0.05,122}=1.97$} \\
\hline & \multicolumn{3}{|c|}{ Abel et al. (1982) } & \multicolumn{3}{|c|}{ Smoorenburg et al. (1986) } \\
\hline & Study \# & $\mathrm{N}$ & & Study \# & $N$ & \\
\hline & 018904 & 45 & & 018906 & 46 & \\
\hline Frequency & Mean & $\mathrm{SD}$ & $\bar{t}$ & Mean & SD & $t$ \\
\hline 125 & 16.5 & 10.8 & 0.87 & & & \\
\hline 250 & 17.4 & 9.9 & 1.45 & 6.6 & 9.7 & 3.89 \\
\hline 500 & 18.7 & 9.3 & 1.58 & 77. & 12.5 & 3.44 \\
\hline 1000 & 20.9 & 9.8 & 1.76 & 90 & 12.9 & 4.05 \\
\hline 2000 & 23.2 & 9.3 & 0.67 & 19.4 & 14.9 & 2.34 \\
\hline 4000 & 28.8 & 9.6 & 0.68 & 24.2 & 14.1 & 2.71 \\
\hline \multirow[t]{5}{*}{8000} & & & & 162 & 15.0 & 4.59 \\
\hline & \multicolumn{3}{|l|}{$t_{0.05,139}=1.97$} & \multicolumn{3}{|l|}{$t_{0.05 .140}=1.97$} \\
\hline & \multicolumn{3}{|c|}{ P-Vermeer et al. (1993) } & & & \\
\hline & Study \# & $N$ & & & & \\
\hline & 018919 & 12 & & & & \\
\hline Frequency & Mean & SD & $\bar{t}$ & & & \\
\hline 125 & 7.9 & 8.4 & 1.95 & & & \\
\hline 250 & 8.3 & 10.7 & 1.76 & & & \\
\hline 500 & 9.2 & 11.8 & 1.63 & & & \\
\hline 1000 & 9.6 & 6.7 & 2.41 & & & \\
\hline 2000 & 15.4 & 6.7 & 2.97 & & & \\
\hline 4000 & 196. & 13.4 & 3.02 & & & \\
\hline 8000 & 17.1 & 13.4 & 2.32 & & & \\
\hline & $t_{0.05 .106}=1.98$ & & & & & \\
\hline
\end{tabular}

and Roberts, 1983; Hempstock and Hill, 1990; Mendez et al., 1986; Padilla, 1976; Pekkarinen, 1987; Pfeiffer et al., 1989; Royster et al., 1991; Passchier-Vermeer et al., 1993; and Smoorenburg et al., 1986). The total data base of 22 studies comprises results from over 90 different industries, in seven countries (Argentina, Canada, Finland, Germany, Netherlands, UK, and U.S.) with a total of approximately 2900 subjects. Of those studies, 16 included data on the four HPDs which were tested in the interlaboratory comparison. For additional details on the studies, readers are referred to the individual reports and to the complete summary by Berger et al. (1996).

Measurements in the field studies were conducted by independent researchers, government-sponsored investigators, and by staff employed at the industries which supplied the data. In all cases, the test subjects were private-sector 
TABLE IV. Bilsom UF-1 earmuff. Mean subject-fit (SF) data from the interlaboratory study, manufacturer's labeled values, and 3 real-world studies. $N$ is number of subjects, SD is standard deviation, shaded values are significantly less than Interlab values at $p<0.05$, and underlined values are significantly greater at $p<0.05$.

\begin{tabular}{|c|c|c|c|c|c|c|}
\hline & $\begin{array}{c}\text { Interlab } \\
\text { Subject Fit }\end{array}$ & $\begin{array}{l}N \\
96\end{array}$ & & $\begin{array}{l}\text { Mfg.'s } \\
\text { Labeled }\end{array}$ & $\begin{array}{l}N \\
10\end{array}$ & \\
\hline Frequency & Mean & $\mathrm{SD}$ & & Mean & $\mathrm{SD}$ & $t$ \\
\hline 125 & 7.4 & 3.6 & & 17.1 & 1.9 & 8.37 \\
\hline 250 & 14.0 & 3.4 & & 19.9 & 1.3 & 5.43 \\
\hline 500 & 20.7 & 3.3 & & 25.6 & 2.4 & 4.56 \\
\hline 1000 & 29.2 & 3.8 & & $\underline{32.8}$ & 1.7 & 2.96 \\
\hline 2000 & 31.7 & 4.2 & & $\overline{40.3}$ & 1.5 & 6.41 \\
\hline 4000 & 35.6 & 4.0 & & 46.7 & 1.4 & 8.69 \\
\hline 8000 & 34.8 & 4.9 & & $\overline{43.9}$ & 2.8 & 5.76 \\
\hline \multicolumn{7}{|c|}{$t_{0.05 .104}=1.98$} \\
\hline & \multicolumn{3}{|c|}{ Hachey/Roberts (1983) } & \multicolumn{3}{|c|}{ Casali/Park (1991) } \\
\hline & Study \# & $\mathbf{N}$ & & Study \# & $\mathbf{N}$ & \\
\hline & 056403 & 31 & & 056416 & 10 & \\
\hline Frequency & Mean & SD & $\bar{t}$ & Mean & SD & $t$ \\
\hline 125 & 7.8 & 4.1 & 0.52 & 8.3 & 4.2 & 0.74 \\
\hline 250 & 8.8 & 5.0 & 6.55 & 12.2 & 4.4 & 1.55 \\
\hline 500 & 168 & 6.2 & 4.51 & 19.3 & 5.2 & 1.20 \\
\hline 1000 & 27.6 & 4.4 & 1.96 & 26.5 & 6.6 & 1.97 \\
\hline 2000 & 30.5 & 6.3 & 1.21 & 262 & 5.5 & 3.82 \\
\hline 4000 & 295 & 9.2 & 5.18 & 36.0 & 6.6 & 0.28 \\
\hline \multirow[t]{5}{*}{8000} & 240 \% & 8.6 & 8.71 & 35.3 & 7.0 & 0.29 \\
\hline & \multicolumn{3}{|l|}{$t_{0.05,125}=1.98$} & \multicolumn{3}{|l|}{$t_{0.05,104}=1.98$} \\
\hline & \multicolumn{3}{|c|}{ Casali/Park (1991) } & & & \\
\hline & Study \# & $\mathbf{N}$ & & & & \\
\hline & 056417 & 10 & & & & \\
\hline Frequency & Mean & SD & $t$ & & & \\
\hline 125 & 9.4 & 2.5 & 1.71 & & & \\
\hline 250 & 13.9 & 2.8 & 0.09 & & & \\
\hline 500 & 21.0 & 2.8 & 0.28 & & & \\
\hline 1000 & 27.3 & 3.7 & 1.51 & & & \\
\hline 2000 & 28.6 & 3.8 & 2.24 & & & \\
\hline 4000 & 384 & 5.2 & 2.05 & & & \\
\hline 8000 & 36.0 & 5.6 & 0.73 & & & \\
\hline & $t_{0.05,104}=1.9$ & & & & & \\
\hline
\end{tabular}

workers or military personnel exposed to noise who were tested in most cases while wearing their own HPDs.

The facilities that have been examined most likely represent the better hearing conservation programs in existence. This presumption is based upon the increased likelihood of finding higher-quality programs among companies and organizations interested in and choosing to participate in the complicated, time consuming, and costly research of the type required for real-world evaluations. In fact, in at least two of the more recent studies, the locations were selected specifically because the authors believed them to be exemplary (Edwards and Green, 1987; Pfeiffer et al., 1989).

Due to the variety of authors who have been involved and the diversity of countries in which the research has been conducted, the real-world data base spans a number of different procedures. Some of the most interesting parameters that could potentially influence the data and are germane to the analyses of this paper include: how the participation of the subjects was arranged (candid versus scheduled testing), and how the attenuation was measured (REAT using large circumaural earcups versus REAT in a small test booth). Berger et al. (1996) in their comprehensive review paper examined these aspects and others, and concluded that within the limits of the variability of the available data, it was appropriate to collapse the results across scheduling method, and across methods of measuring attenuation as well.

A very recent evaluation of real-world hearing protector performance was also reviewed for this paper (Scott, 1995), but not included in the data tables. This extensive study of 350 subjects at 9 sites included E-A-R Classic foam earplugs, one of the four HPDs evaluated in this study, and the one for which substantial data already existed. Although the Scott results were not available in time for full inclusion in this study and statistical analyses, qualitative comparison to the 16 existing measurements on the foam plug indicated that the newer values only served to confirm that which had already been observed.

The real-world data for the four HPDs of this study are presented in Tables I-IV, along side the previously mentioned interlaboratory results. A blank cell indicates the authors did not test attenuation at that frequency, most notable being Padilla (1976) who only tested at $500 \mathrm{~Hz}$. For each study, the reference is provided (see the references), as is the number of employees who were tested, and the study identification number (for the author's internal purposes).

\section{Analysis}

It was not possible to use a statistical tool such as an ANOVA to provide a basis for comparison of the real-world (RW), interlaboratory subject-fit (SF), and manufacturers' published (MFG) data, since raw RW and MFG values were not available. However, when the means and variances of two populations are compared with the assumption being that the two populations will have equal means and variances, $\mu_{1}=\mu_{2}$ and $\left(\sigma_{1}\right)^{2}=\left(\sigma_{2}\right)^{2}=\sigma^{2}$, the distribution is that of the $t$-test (Mendenhall, 1975). The $t$-tests were run for independent samples of differing size, with a presumption of normal distributions and equal variances in both samples. The equal-variance assumption was appropriate for the SFto-RW comparisons, but not for the SF-to-MFG comparisons, since in the latter case the MFG variances were substantially smaller. The effect of the inhomogeneity of variance in this instance, wherein the distribution with the lesser variance also has less than or equal to the sample size of the other distribution, is to make the $t$-test more conservative, i.e., it is less likely to reach significance. In spite of this, all SF-to-MFG differences were found to be significantly different.

The computed $t$-values are also listed in Tables I-IV, along with the associated degrees of freedom indicated in the subscript. The mean RW values which are significantly less than the SF values (at $p<0.05$ ) are shaded, and those which are significantly greater are underlined.

\section{RESULTS}

The product for which the greatest amount of data are available is the E-A-R ${ }^{\circledR}$ Classic foam earplug (see Table I and Fig. 1). There are 16 separate measurements from 11 different reports with a total subject count of 633 . For $61 \%$ of the 108 possible $t$-test comparisons the SF values are statistically greater than the RW data, $28 \%$ of the time there 


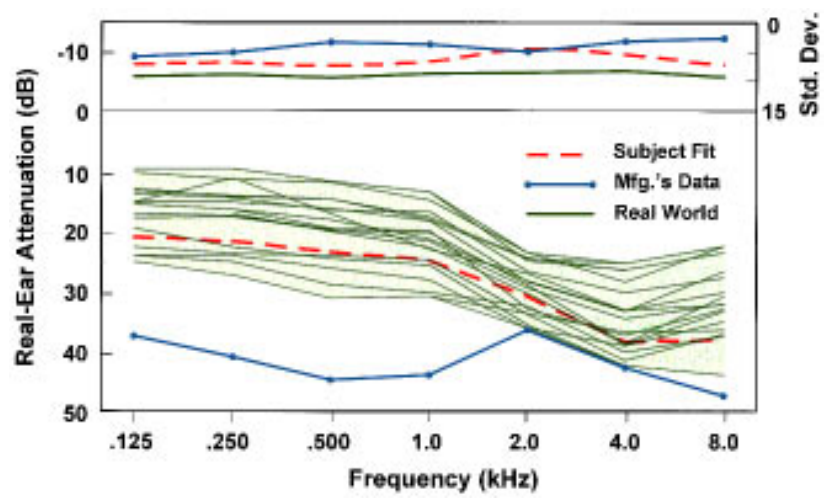

FIG. 1. E-A-R ${ }^{\circledR}$ Classic foam earplug: Mean subject-fit (SF) data from interlaboratory study and mfg.'s labeled values compared to 16 real-world studies. Individual real-world studies shown by thin green lines w/out symbols; bold green line w/out symbols is avg. real-world standard deviation and shading shows range of real-world data.

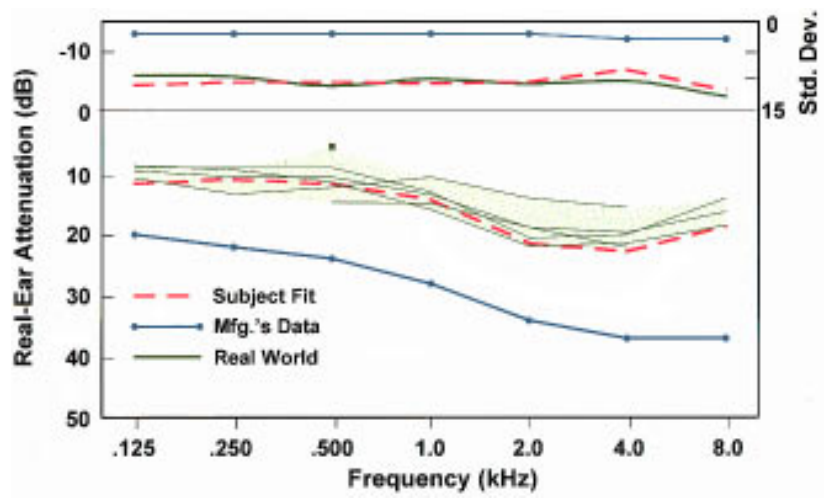

FIG. 2. V-51R earplug: Mean subject-fit (SF) data from interlaboratory study and mfg.'s labeled values compared to five real-world studies. Individual real-world studies shown by thin green lines w/out symbols and by filled box; bold green line w/out symbols is avg. real-world standard deviation and shading shows range of real-world data.

were no significant differences, and only $11 \%$ of the time did the RW values exceed the SF values. Stated alternatively, in $89 \%$ of the comparisons the SF values equaled or exceed the RW data. This can be appreciated visually in Fig. 1, wherein it is clear the SF data represent approximately the upper quartile of the field values. In all cases the MFG data were statistically greater than the SF values, and in the figure the MFG values can be seen to be well outside the range of field data, except at 2 and $4 \mathrm{kHz}$.

The data for the remaining two earplugs tell a similar story (Tables II and III, and Figs. 2 and 3), although the fact that fewer field studies are available for examination makes it difficult to ascertain whether the SF data represent an upper bound, an upper quartile, or some other value. There are five studies of the V-51R earplug with a total subject count of 308, although 183 of those subjects were from one study (Padilla, 1976) who only measured attenuation at $500 \mathrm{~Hz}$, albeit a frequency that has been shown to be an excellent indicator of overall protection (Berger, 1989). For 20\% of the 25 possible $t$-test comparisons the SF values were statistically greater than the RW data, and in the remaining $80 \%$ there were no significant differences. In no instances did the field values significantly exceed the SF measurements.

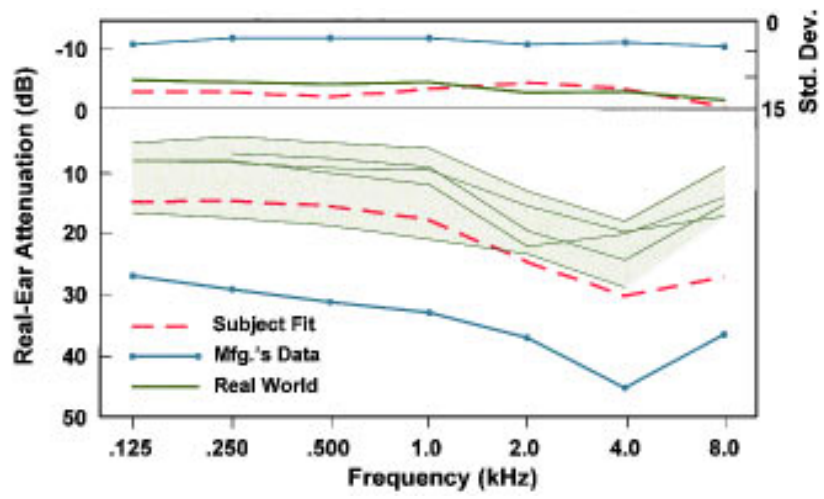

FIG. 3. EP100 earplug: Mean subject-fit (SF) data from interlaboratory study and mfg.'s labeled values compared to five real-world studies. Individual real-world studies shown by thin green lines w/out symbols; bold green line w/out symbols is avg. real world standard deviation and shading shows range of real-world data.

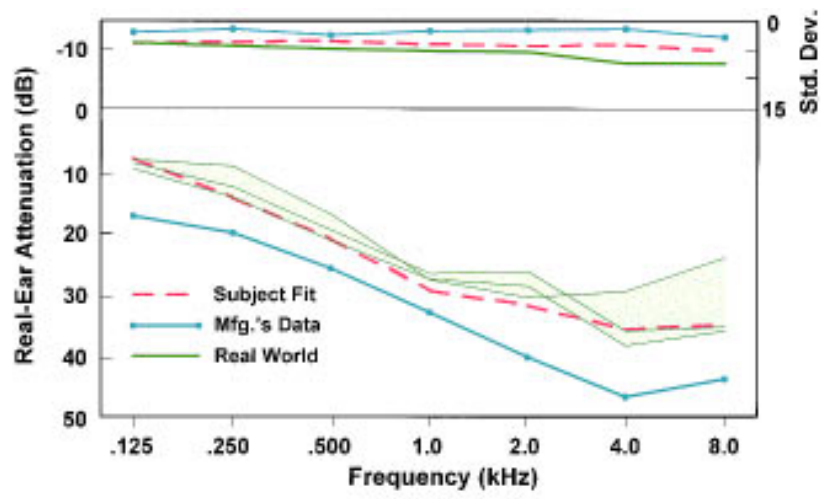

FIG. 4. UF-1 earmuff: Mean subject-fit data (SF) from interlaboratory study and mfg.'s labeled values compared to three real-world studies. Individual real-world studies shown by thin green lines w/out symbols; bold green line w/out symbols is avg. real world standard deviation and shading shows range of real-world data.

There are also five studies of the EP100 earplug, with a total subject count of 153 . For $67 \%$ of the 33 possible $t$-test comparisons the SF values are greater than the RW data, and in the remaining 33 of the comparisons there were no differences. In no instances did the field values significantly exceed the SF measurements. For both the V-51R and the EP100, the MFG data significantly exceeded the SF data at all frequencies.

With earmuffs, the expectation was that there would be less difference between the lab and field data since there is less to go wrong with the fitting of earmuffs under field conditions and since there is also less potential for the experimenter to "over fit" the earmuff for high attenuation in the laboratory setting. However, the reduced variance of the earmuff data caused smaller measured differences to reach significance. There were three separate measurements of the UF-1 earmuffs from two different reports with a total subject count of 51, and the values may be found in Table IV and are plotted in Fig. 4. For 33\% of the 21 possible $t$-test comparisons the SF values are greater than the RW data, and in the remaining $67 \%$ there were no significant differences. In no instances did the field values significantly exceed the SF measurements. As noted with all of the other products, the 
MFG data significantly exceeded the SF data at all frequencies.

\section{DISCUSSION}

In order to create a procedure that generates "valid" data, the question of course has to be asked, "Valid with respect to what?' In practice, a wide range of HPD attenuation values may be observed in the workplace, from essentially no attenuation at all for devices poorly fitted by untrained users who incorrectly and inconsistently wear their HPDs, to much higher levels of protection that may be obtained under ideal conditions in workplaces with the most successful hearing conservation programs. It makes no sense to excessively derate hearing protector performance to estimate worst-case attenuation values, since worst-case values are much more heavily influenced by factors other than the hearing protectors themselves, such as substantial misuse of products. Neither is it appropriate to utilize optimum laboratory-fit values to estimate field performance, since such laboratory-based values are in essence estimates of idealized protection obtained under pristine conditions and/or performance attained by unusually well-trained and motivated users.

In developing a procedure to estimate field performance, the decision was made by the Working Group to attempt to approximate "achievable", results. Such results were defined as among the higher values of attenuation attained by groups of informed users in well-managed industrial and military hearing conservation programs. The validity of the estimates was assessed and substantiated by the analyses in this report, and by prior analyses (Franks and Casali, 1993).

Ideally, the approach to reduction of laboratory versus real-world discrepancies would be to improve field performance to match laboratory data, keeping in mind that under no circumstances can one hope to duplicate optimum laboratory data for groups of users under field conditions. Regardless, most agree that industrial hearing conservation practice must be enhanced so that better real-world HPD performance can be realized (Berger, 1992). However, it is also clear that a laboratory method of measuring hearing protector attenuation that yields data which more closely correlate with existing, or even potential field performance, would be a valuable predictive tool. The development of such a tool has been one of the principal goals of Working Group 11.

The assessment of the degree to which the Working Group achieved its goal was based on comparison of the laboratory data measured via use of the new proposed protocol, to field performance data. The field data were taken from a wide range of available studies. Although some would argue that certain of the real-world studies might represent less than ideal field practice, the general agreement across studies, as well as the reasoning expressed earlier in this paper regarding the types of companies and hearing conservation programs that would be likely to participate, strongly argue that the current real-world data base provides a favorable representation of hearing conservation practice in the latter part of the twentieth century. As such, the fact that the laboratory-based data from the protocol proposed herein predicts the upper bounds of field-measured hearing protec- tor attenuation is, in these authors' opinion, a reasonable measure of the suitably of that protocol. This suggests that the laboratory data are achievable by groups of users in the field, but are not guaranteed. Certainly, sincerely interested and/or highly motivated individual users may exceed these values (and of course others will fall short), but the purpose of such laboratory-based data is to provide a statistical indicator, not an absolute guarantee, of what hearing conservationists can expect to attain in an overall hearing conservation program. How the mean laboratory data are adjusted by the subtraction of one or more standard deviations in order to reflect what $84 \%$, or $98 \%$, or some other proportion of the users will achieve, is up to those who regulate safety or who implement programs based on these data (Berger and Royster, 1996).

With this discussion in mind, the results of the analyses provide strong support for the use of SF data, and a strong contraindication for use of existing U.S. laboratory-based test data for the estimation of field performance. The SF data were shown to provide essentially an upper-bound estimate for the premolded earplugs and the earmuff, and something closer to an upper-quartile estimate for the foam earplug. One could argue that the SF data provide too high a prediction of RW attenuation, but certainly not one that is too low. However, with the precision that is available in subjective testing of this nature, the Working Group agreed that the appropriate balance between over- and underestimation of field performance had been achieved. The MFG data, based on EPA-required testing using the 1974 standard were shown to always significantly exceed SF data, and to also always exceed field performance data by a substantial amount.

It is concluded that the data that results from the subjectfit method of Working Group 11, as implemented in the recently approved standard S12.6-1997, provide an improved estimate of the field performance of HPDs. Furthermore, this estimate represents the upper range of attenuation values which are achievable by groups of users in well-managed and well-supervised industrial and military hearing conservation programs.

\section{ACKNOWLEDGMENTS}

This study would not have been possible without the careful, patient, and dedicated efforts of those individuals who collected and recorded the interlaboratory subject-fit data, and maintained cheerful rapport with the subjects throughout the process. Each of these individuals is gratefully acknowledged for this considerable effort: at Armstrong Laboratory, U. S. Air Force personnel Captain Nancy Green and Captain Denise West; at NIOSH Taft Laboratories, Curtis W. Sizemore; and at U. S. Army Aeromedical Research Laboratory, Barbara A. Murphy. Edward F. Krieg of NIOSH is acknowledged for his assistance with statistical analysis of the data.

Abel, S. M., Alberti, P. W., and Riko, K. (1982). “User fitting of hearing protectors: Attenuation results,' in Personal Hearing Protection in Industry, edited by P. W. Alberti (Raven, New York), pp. 315-322. 
ANSI (1974). S3.19-1974, “American National Standard Method for the measurement of real-ear protection of hearing protectors and physical attenuation of earmuffs" (American National Standards Institute, New York).

ANSI (1984). S12.6-1984, "American National Standard Method for the measurement of the real-ear attenuation of hearing protectors" (American National Standards Institute, New York).

ANSI (1997). S12.6-1997, “'American National Standard Methods for measuring real-ear attenuation of hearing protectors"' (American National Standards Institute, New York).

Behar, A. (1985). "Field evaluation of hearing protectors,' Noise Control Eng. J. 24, 13-18.

Berger, E. H. (1988). "Can real-world hearing protector attenuation be estimated using laboratory data?,' J. Sound Vib. 22, 26-31.

Berger, E. H. (1989). "Exploring procedures for field testing the fit of earplugs,' in Proceedings, 1989 Industrial Hearing Conservation Conference, Off. Eng. Serv. (University of Kentucky, Lexington, KY), pp. 7-10.

Berger, E. H. (1992). "'Development of a laboratory procedure for estimation of the field performance of hearing protectors," in Proceedings, Hearing Conservation Conference, Off. Eng. Serv. (University of Kentucky, Lexington, KY), pp. 41-45.

Berger, E. H., Franks, J. R., and Lindgren, F. (1996). "International review of field studies of hearing protector attenuation," in Scientific Basis of Noise-Induced Hearing Loss, edited by A. Axelsson, H. Borchgrevink, R. P. Hamernik, P. Hellstrom, D. Henderson, and R. J. Salvi (Thieme Medical, New York), pp. 361-377.

Berger, E. H., and Kieper, R. W. (1991). "Measurement of the real-world attenuation of E-A-R ${ }^{\circledR}$ foam and Ultrafit ${ }^{\circledR}$ brand earplugs on production employees,', E-A-R Tech. Rept. 91-30/HP, Indianapolis, IN.

Berger, E. H., and Royster, L. H. (1996). "In search of meaningful measures of hearing protector effectiveness,' Spectrum Suppl. 1, 13, 29.

Casali, J. G., and Epps, B. W. (1986). 'Effects of user insertion/donning instructions on noise attenuation of aural insert hearing protectors,' Hum. Factors 28, 195-210.

Casali, J. G., and Park M. Y. (1991). "Laboratory vs field attenuation of selected hearing protectors,' Sound and Vibration 25(10), 28-38.

Chung, D. Y., Hardie, R., and Gannon, R. P. (1983). "The performance of circumaural hearing protectors by dosimetry,' J. Occup. Med. 15, 679682

Crawford, D. R., and Nozza, R. J. (1981). "Field performance evaluation of wearer-molded ear inserts,', presented at Am. Ind. Hyg. Conf., Abstract \#398, Portland, OR.

Durkt, Jr., G. (1993). "Field evaluations of hearing protection devices at surface mining environments," Mine Safety and Health Admin., IR 1213, Pittsburgh, PA.

Edwards, R. G., Broderson, A. B., Green, W. W., and Lempert, B. L. (1983). "A second study of the effectiveness of earplugs as worn in the workplace,' Noise Control Eng. J. 20, 6-15.

Edwards, R. G., and Green, W. W. (1987). "Effect of an improved hearing conservation program on earplug performance in the workplace,' Noise Control Eng. J. 28, 55-65.

Edwards, R. G., Hauser, W. P., Moiseev, N. A., Broderson, A. B., and
Green, W. W. (1978). "Effectiveness of earplugs as worn in the workplace,' Sound and Vibration 12(1), 12-22.

EPA. (1979). "Noise labeling requirements for hearing protectors," U. S. Environmental Protection Agency, Fed. Regist. 44(190), 40CFR Part 211, 56130-56147.

Fleming, R. M. (1980). “A new procedure for field testing of earplugs for occupational noise reduction,"' Doctoral Thesis at Harvard School of Public Health, Boston, MA.

Franks, J. R., and Casali, J. G. (1993). "Hearing protector attenuation from subject-fit methods at the work site and in the laboratory,', J. Acoust. Soc. Am. 94, 1791-1792.

Goff, R. J., and Blank, W. J. (1984). "A field evaluation of muff-type hearing protection devices,' Sound and Vibration 18(10), 16-22.

Hachey, G. A., and Roberts, J. T. (1983). "Real world effectiveness of hearing protection,'” presented at Am. Ind. Hyg. Conf., Abstract \#462, Philadelphia, PA.

Hempstock, T. I., and Hill, E. (1990). "The attenuations of some hearing protectors as used in the workplace,' Ann. Occup. Hyg. 34, 453-470.

Mendenhall, W. (1975). Introduction to Probability and Statistics, 4th ed. (Duxbury Press, North Scituate, MA), pp. 222-224.

Mendez, A., Salazar, E., and Bontti, H. (1986). "Attenuation measurement of hearing protectors in workplace,' 12 th Int. Congr. on Acoustics, Toronto, Vol. 1, paper B10-2.

Padilla, M. (1976). "Ear plug performance in industrial field conditions," Sound and Vibration 10(5), 33-36

Passchier-Vermeer, W., van den Berg, R., and Crijns, H. (1993). "Development of a simplified attenuation test method for personal hearing protection devices and for determining the attenuation values in real working situations,', TNO, NIPG Pub. No. 93.004, The Netherlands.

Pekkarinen, J. (1987). "Industrial impulse noise, crest factor and the effects of earmuffs,' Am. Ind. Hyg. Assoc. J. 48, 861-866.

Pfeiffer, B. H., Kuhn, H-D., Specht, U., and Knipfer, C. (1989). "'Sound attenuation by hearing protectors in the real world (in German),' Berufsgenossenschaftliches Institut fur Arbeitssicherheit Report 5/89, West Germany.

Regan, D. E. (1975). "Real ear attenuation of personal ear protective devices worn in industry,', Doctoral Thesis at Kent State University, University Microfilms Int., Ann Arbor, MI.

Royster, J. D., Ostendorf, J. S., Royster, L. H., and Berger, E. H. (1991). Personal communication.

Royster, J. D., Berger, E. H., Merry, C. J., Nixon, C. W., Franks, J. R., Behar, A., Casali, J. G., Dixon-Ernst, C., Kieper, R. W., Mozo, B. T., Ohlin, D., and Royster, L. H. (1996). "Development of a new standard laboratory protocol for estimating the field attenuation of hearing protection devices. Part I. Research of Working Group 11, Accredited Standards Committee S12, Noise," J. Acoust. Soc. Am. 99, 1506-152.

Scott, R. F. (1995). "Study of factors affecting at-work protection afforded by hearing protection devices on steelworks,', British Steel plc, Doc. FR S373-5 942, London, UK.

Smoorenburg, G. F., ten Raa, B. H., and Mimpen, A. M. (1986). "Realworld attenuation of hearing protectors,' 12th Int. Congr. on Acoustics, Toronto, Vol. 1, paper B9-6. 